\title{
On the Extension of Real Places
}

ManfRed Knebusch 


\title{
On the Extension of Real Places ${ }^{1}$ )
}

\author{
MANFRED KNEBUSCH
}

\section{Introduction}

About twenty years ago S. Lang studied places $\varphi: K \rightarrow R \cup \infty$ on a field $K$ with values in a fixed real closed field $R$ ([L]). One of his main results was the theorem, that any such place $\varphi$ can be extended to an $R$-valued place on a suitable real closure of $K$ ([L], Th. 6). Now the real closures of $K$ correspond up to $K$-isomorphisms uniquely to the (total) orderings of $K$. Thus one may ask whether it is possible to obtain a more precise version of Lang's theorem by a more thorough analysis of the relations between orderings and real places. This question is the starting point of the present paper.

We say that an ordering $\alpha$ of $K$ lies over the place $\varphi: K \rightarrow R \cup \infty$ or that $\varphi$ and $\alpha$ are compatible, if any element $a$ of $K$ which is positive with respect to $\alpha$ has value $\varphi(a)=\infty$ or $\varphi(a) \geqslant 0$. (Recall that $R$ is ordered in a unique way). In $\S 1$ we first show that over any real place $\varphi$ lies at least one ordering $\alpha$. Then we prove the following refinement of Lang's theorem:

THEOREM 1.6. Assume that $L$ is an algebraic field extension of $K$, that $\beta$ is an ordering on $L$ and that $\varphi$ is an $R$-valued place on $K$, compatible with the restriction of $\beta$ to $K$. Then there exists a unique $R$-valued place $\psi$ on $L$ extending $\varphi$ and compatible with $\beta$.

Harrison ([H]), and Leicht, Lorenz ([LL]) showed that the orderings $\alpha$ of a field $K$ correspond uniquely to the signatures $\sigma$ of $K$, i.e. the ring homomorphisms $\sigma: W(K)$ $\rightarrow \mathbf{Z}$, where $W(K)$ denotes the Witt ring of non singular symmetric bilinear forms over $K$ ([W]). As usual we denote for any $a \neq 0$ in $K$ by $(a)$ the element of $W(K)$ represented by the form $B: K \times K \rightarrow K, B(x, y)=a x y$. The signature $\sigma$ corresponding to the ordering $\alpha$ is characterized by $\sigma(a)=+1$ if $a>0$ with respect to $\alpha$, and $\sigma(a)=-1$ if $a<0$. (Recall that $W(K)$ is generated by the elements $(a)$.) We shall make strong use of this connection between orderings and Witt rings, and we shall always identify an ordering $\alpha$ with the corresponding signature $\sigma$. The unique signature $W(R) \rightarrow \mathbf{Z}$ will be denoted by $\varrho$.

As will be explained in $\S 2$, any $R$-valued place $\varphi$ on $K$ yields a well defined additive map $\varphi_{*}: W(K) \rightarrow \mathbf{Z}$, whose value on an element $(a)$ is obtained in the following way: If the square class $a K^{* 2}$ contains an element $a^{\prime}=a b^{2}$ such that $\varphi\left(a^{\prime}\right) \neq 0$ and $\neq \infty$, then $\varphi_{*}(a)=\varrho\left(\varphi\left(a^{\prime}\right)\right)$ with an arbitrary choice of $a^{\prime}$. If $a K^{* 2}$ contains no such elements,

1) The main results of this paper have been announced in $\left[K_{2}\right.$, part $\left.B\right]$. 
then $\varphi_{*}(a)=0$. Obviously a signature $\sigma$ lies over $\varphi$ if and only if $\sigma(a)=\varphi_{*}(a)$ for all $a$ in $K^{*}$ such that $\varphi_{*}(a) \neq 0$.

We prove in $\S 2$ the following counterpart of the theorem above:

THEOREM 2.6. Assume that $L$ is an arbitrary field extension of $K$, that $\psi$ is an $R$-valued place on $L$ and that $\sigma$ is a signature of $K$ lying over $\psi \mid K$. There exists a signature $\tau$ of $L$ lying over $\psi$ and extending $\sigma\left\{\right.$ i.e. $\tau(a)=\sigma(a)$ for all $a$ in $\left.K^{*}\right\}$ if and only if $\sigma(a)=\psi_{*}(a)$ for all $a$ in $K^{*}$ with $\psi_{*}(a) \neq 0$.

We further prove in $\S 2$ a theorem about the real places in the field composites of an algebraic extension $L_{1} / K$ and an arbitrary extension $L_{2} / K$.

Our work in $\S 3$ originates from the question, how many $R$-valued extensions has a given $R$-valued place $\varphi$ of $K$ in a finite field extension $L / K$. Recall that the regular trace $\operatorname{Tr}_{L / K}$ induces an additive map $\operatorname{Tr}_{L / K}^{*}: W(L) \rightarrow W(K)$ mapping the class of a symmetric bilinear space $(E, B)$ over $L$ to the class of the space $\left(E, \operatorname{Tr}_{L / K^{\circ}} B\right)$ over $K$ (cf $[\mathrm{S}])$. We prove the following trace formula: For any $x$ in $W(L)$

$$
\varphi_{*}\left(\operatorname{Tr}_{L / K}^{*}(x)\right)=\sum_{\psi \uparrow \varphi} \psi_{*}(x)
$$

where $\psi$ runs through all $R$-valued places of $L$ extending $\varphi$, with the convention that the right hand side is zero if there are no such places $\psi$. Applying this formula to the unit element (1) of $W(L)$ one obtains that $\varphi$ has exactly $\varphi_{*}\left(\operatorname{Tr}_{L / K}^{*}(1)\right) R$-valued extensions to $L$.

The final section 4 gives an application of the theorem 1.6 cited above to the problem of extending an $R$-valued place $\varphi$ on $K$ to a field $L$ which is finitely generated over $K$ but not necessarily algebraic.

To prevent misunderstandings I remark that in this paper different places with the same valuation ring are never identified and that a place is allowed to be trivial, i.e. to avoid the value $\infty$.

\section{$\S 1$}

We first recall some well known facts and notations (cf [L], [AS]). Assume that on a field $K$ an ordering $\sigma$ is given and that $k$ is a subfield of $K$. An element $a$ of $K$ is called infinitely large over $k$ (with respect to $\sigma$ ) if there is no element $c>0$ in $k$ such that $|a|<c$. Here $|a|$ denotes the element $a$ if $a \geqslant 0$ and $-a$ if $a \leqslant 0$ with respect to $\sigma$. The set of elements of $K$, which are not infinitely large over $k$, is a valuation ring of $K$ ([AS], p. 95) which we call the valuation ring $\mathfrak{D}=\mathfrak{D}(K / k, \sigma)$ associated with $\sigma$ over $k$. Obviously the maximal ideal $\mathfrak{m}$ of $\mathfrak{b}$ is the set of all elements $a$ in $K$ which are infinitely small over $k$, i.e. $|a|<c$ for all $c>0$ in $k$.

Clearly $k \subset \mathfrak{D}$. A field $k^{\prime}$ with $k \subset k^{\prime} \subset K$ is called archimedian over $k$ (with respect 
to $\sigma$ ) if $k^{\prime} \subset \mathfrak{D}$. Then $\mathfrak{D}$ is also the valuation ring associated with $\sigma$ over $k^{\prime}$. It is clear from general valuation theory that any algebraic extension $k^{\prime}$ of $k$ in $K$ is archimedian over $k$.

As follows from Zorn's lemma, there exists at least one intermediate field $\tilde{k} \supset k$ which is maximal archimedian over $k$, i.e. $\tilde{k}$ is archimedian over $k$, but no field $k^{\prime} \supset \tilde{k}$ different from $\tilde{k}$ is archimedian over $k$ ([L], p. 379). We say that $k$ is maximal archimedian in $K$, if $k=\tilde{k}$. It is clear from general valuation theory that the field $\mathrm{o} / \mathrm{m}$ is always algebraic over $\tilde{k}$.

The ordering $\sigma$ of $K$ induces an ordering $\bar{\sigma}$ of $\mathfrak{v} / \mathrm{m}$, characterized in the following way ([AS], p. 95): An element $\bar{a}$ of $\mathfrak{p} / \mathrm{m}$ is positive if and only if a preimage $a$ in $\mathfrak{v}$ is positive. (It does not matter which preimage is chosen.) If $K$ is real closed then $\tilde{k}$ is real closed, since $\tilde{k}$ is algebraically closed in $K$. Thus in this case $\tilde{k}$ maps bijectively onto $\mathfrak{v} / \mathrm{m}$ ([AS], p. 95).

All rings in this paper are commutative and have a unit element and all ring homomorphisms map 1 to 1 . The unit group of a ring $A$ is denoted by $A^{*}$. We further denote by $W(A)$ the Witt ring of non degenerate symmetric bilinear forms over $A$, and for any homomorphism $\alpha: A \rightarrow C$ into a ring $C$ we denote by $W(\alpha)$ the corresponding ring homomorphism from $W(A)$ to $W(C)$. We refer the reader to [K], [KRW, $\S 1$ ], or [M] for these notions. For any element $a$ in $A$ we denote by $(a)$ the element of $W(A)$ which is represented by the form $B: A \times A \rightarrow A, B(x, y)=a x y$. These elements $(a)$ form a subgroup $Q(A)$ of $W(A)$, which will be identified with the group $A^{*} / A^{* 2}$ of square classes. If $A$ is local, i.e. $A$ has only one maximal ideal, the $\operatorname{ring} W(A)$ is generated by $Q(A)$ (e.g. [KRW, $\S 1]$ ). In this paper only the Witt rings of fields and valuation rings will play a rôle.

As explained in the introduction, the signatures $\sigma$ of a field $K$, i.e. the homomorphisms $\sigma$ from the ring $W(K)$ to $\mathbf{Z}$, correspond uniquely to the orderings of $K$. Let $L$ be a field extension of $K$ and $i$ denote the inclusion map from $K$ into $L$. For any signature $\tau$ of $L$ we denote by $\tau \mid K$ the signature $\sigma=\tau \circ W(i)$ of $K$, and we say that $\sigma$ is the restriction of $\tau$ to $K$, or that $\tau$ is an extension of to $L$. This terminology is compatible with the usual meaning of extension and restriction of orderings.

Throughout this paper $R$ denotes a real closed field and $\varrho$ denotes the signature of $R$. For a moment we forget about orderings of the field $K$ and consider a place $\varphi: K \rightarrow R \cup \infty$. Let $\mathfrak{D}$ denote the valuation ring of $\varphi$, i.e. the ring of all elements $x$ in $K$ with $\varphi(x) \neq \infty$. By composing the map $W(\varphi \mid \mathfrak{o})$ from $W(\mathfrak{D})$ to $W(R)$ with $\varrho: W(R)$ $\neg \mathbf{Z}$ we obtain a ring homomorphism from $W(\mathfrak{D})$ to $\mathbf{Z}$ which we denote by $\hat{\varphi}$. On a generator $(a)$ of $W(\mathfrak{D}), a$ in $\mathfrak{D}^{*}$, the map takes the value 1 if $\varphi(a)>0$ and -1 if $\varphi(a)<0$.

Since $\mathfrak{o}$ is a Prüfer ring, the map $W(i): W(\mathfrak{b}) \rightarrow W(K)$, obtained from the inclusion map $i: \mathrm{o} \rightarrow K$ is injective ([K, Satz 11.1.1]; the reader may also consult $\left[\mathrm{KRW}_{1}\right.$, Lemma 1.1] or [M, p. 93], where this fact is stated for Dedekind rings but proved for Prüfer rings). We shall always consider $W(\mathrm{D})$ as a subring of $W(K)$. 
We say that a signature $\sigma$ of $K$ lies over $\varphi$, or that $\varphi$ is compatible with $\sigma$, if $\sigma$ extends $\hat{\varphi}$. Obviously this definition coincides with the definition given in the introduction.

PROPOSITION 1.1. [ $\mathrm{KRW}_{2}$, 1.13.] Over any place $\varphi: K \rightarrow R \cup \infty$ lies at least one signature $\sigma$ of $K$.

Since this fact is central for the present work, we recall the proof given in $\left[\mathrm{KRW}_{2}\right]$ : The kernel $P$ of $\hat{\varphi}$ is a minimal prime ideal of $W(\mathfrak{o})[\mathrm{KRW}]$. Thus there exists at least one prime ideal $Q$ of $W(K)$ lying over $P$ [B, Chap. II, $\S 2$, no. 6, Prop. 16]. Since $W(\mathfrak{D}) / P \cong \mathbf{Z}$ embeds into $W(K) / Q$ we must have $W(K) / Q \cong \mathbf{Z}$ ([LL], [H]). The only homomorphism $\mathfrak{D}: W(K) \rightarrow \mathbf{Z}$ with kernel $Q$ is the desired signature.

LEMMA 1.2. (cf [L], p. 382) Let $\varphi$ be an $R$-valued place on $K$ and $\sigma$ be a signature of $K$ lying over $\varphi$. Assume further that $a$ and $b$ are elements of $K$ and $\varphi(a) \neq \infty$. Then with respect to the orderings corresponding to $\sigma$ and $\varrho$ the following are true:

(i) $b>a$ implies $\varphi(b)=\infty$ or $\varphi(b) \geqslant \varphi(a)$.

(ii) $0<b<a$ implies $\varphi(b) \neq \infty$ and $0 \leqslant \varphi(b) \leqslant \varphi(a)$.

Proof. (i) $b-a>0$ implies $\varphi(b-a)=\infty$ or $\varphi(b-a) \geqslant 0$ and thus $\varphi(b)=\infty$ or $\varphi(b) \geqslant \varphi(a)$.

(ii) This is clear if $\varphi(b)=0$. Assume now $\varphi(b) \neq 0$. Then $\varphi\left(a b^{-1}\right)=\varphi(a) \varphi\left(b^{-1}\right) \neq \infty$ and we obtain from $a b^{-1}>1$ and (i) that $\varphi(a) \varphi\left(b^{-1}\right) \geqslant 1$. Thus certainly $\varphi(b) \neq \infty$, and we obtain from $b>0$ that $\varphi(b)>0$. Thus $\varphi(a) \geqslant \varphi(b)$. q.e.d.

PROPOSITION 1.3. (cf. [L], Th. 5). Let $\varphi$ be an R-valued place on $K$ and $k$ be a subfield of $K$ on which $\varphi$ is trivial. Assume that $R$ is archimedian over $\varphi(k)$. Then for a signature $\tau$ on $K$ the following are equivalent:

(i) $\tau$ lies over $\varphi$.

(ii) The valuation ring $\mathfrak{D}$ of $\varphi$ coincides with the valuation ring $\mathfrak{D}(K / k, \tau)$ of $\tau$ over $k$. The homomorphism $\bar{\varphi}: \mathrm{D} / \mathrm{m} \rightarrow R$ induced by $\varphi$ on the residue class field of $\sigma$ is order preserving with respect to the ordering $\bar{\tau}$, induced by $\tau$ on $\mathrm{D} / \mathrm{m}$, and $\varrho$.

PROOF. a) Let $\mathfrak{D}^{\prime}$ denote the ring $\mathfrak{D}(K / k, \tau)$ and $\mathrm{m}^{\prime}$ denote the maximal ideal of $\mathfrak{b}^{\prime}$. We first assume $\mathfrak{o}^{\prime}=\mathfrak{D}$ and analyze the situation in this case. That $\bar{\varphi}$ is order preserving with respect to $\bar{\tau}$ and $\varrho$ means in the language of quadratic forms that the map $\varrho \circ W(\bar{\varphi})$ from $W(\mathfrak{v} / \mathrm{m})$ to $\mathbf{Z}$ coincides with $\bar{\tau}$. We denote the canonical map from $\mathfrak{v}$ onto $\mathfrak{D} / \mathfrak{m}$ by $\alpha$. Clearly $\tau \mid W(\mathfrak{o})=\bar{\tau} \circ W(\alpha)$. Now $W(\alpha)$ is surjective, since all generators $(\bar{a}), \bar{a}$ in $(\mathfrak{D} / \mathfrak{m})^{*}$, can be lifted to elements $(a)$ in $W(\mathfrak{D})$. Therefore the equation $\bar{\tau}=\varrho \circ W(\bar{\varphi})$ is equivalent to $\tau \mid W(\mathfrak{o})=\varrho \circ W(\varphi \mid \mathfrak{o})=\hat{\varphi}$. Thus (ii) $\Rightarrow(\mathrm{i})$ is clear, and to prove (i) $\Rightarrow$ (ii) it only remains to be shown that if $\tau$ lies over $\varphi$ the rings $\mathfrak{v}^{\prime}$ and $\mathfrak{v}$ coincide.

b) Assume that $\tau$ lies over $\varphi$. We first show that $\mathfrak{m} \subset \mathfrak{m}^{\prime}$. Let $a$ be an element of $K$ 
which is not in $\mathrm{m}^{\prime}$ and let $b=|a|$ with respect to $\tau$. There exists some $c>0$ in $k$ with $b>c$. By Lemma 1.2(i) we obtain $\varphi(b)=\infty$ or $\varphi(b) \geqslant \varphi(c) \geqslant 0$. Since $\varphi(c) \neq 0$, certainly $\varphi(b) \neq 0$ and thus $\varphi(a) \neq 0$, i.e. $a$ lies not in $m$. This proves $m \subset \mathfrak{m}^{\prime}$.

Now we show $\mathrm{m}^{\prime} \subset \mathfrak{m}$. Then $\mathfrak{v}=\mathfrak{v}^{\prime}$ will be clear. Assume $a$ is an element of $\mathrm{m}^{\prime}$ and without loss of generality $a>0$. For any $c>0$ in $k$ we have $0<a<c$ and thus by Lemma 1.2 (ii) $0 \leqslant \varphi(a) \leqslant \varphi(c)$. Since $R$ is archimedian over $\varphi(k)$ the value $\varphi(a)$ must be zero i.e. $a$ lies in $\mathrm{m}$. q.e.d.

EXAMPLE 1.4. If $k$ is a maximal subfield of $K$ such that a given place $\varphi: K \rightarrow$ $\rightarrow R \cup \infty$ is trivial on $k$ then for any signature $\tau$ of $K$ the conditions (i) and (ii) of Proposition 1.3 are equivalent. In fact, all values of $\varphi$ lie in the algebraic closure $R^{\prime}$ of $\varphi(k)$ in $R$, which is archimedian over $\varphi(k)$. Replace $R$ by $R^{\prime}$ !

COROLLARY 1.5. Let $\sigma$ be a signature on a field $K$ and $k$ be a subfield of $K$, whose algebraic closure $k^{\prime}$ in $K$ is maximal archimedian with respect to $\sigma$. Further assume that $\chi: k \rightarrow R$ is an order preserving homomorphism with respect to $\sigma \mid k$ and $Q$. Then there exists a unique place $\varphi: K \rightarrow R \cup \infty$ with the following properties:

(i) $\varphi$ is compatible with $\sigma$,

(ii) $\varphi \mid k=\chi$,

(iii) $\varphi$ is zero dimensional over $k$, i.e. all values $\neq \infty$ of $\varphi$ are algebraic over $\chi(k)$.

Proof. We replace $R$ by the algebraic closure of $\varphi(k)$ in $R$ and then forget condition (iii). According to Proposition 1.3 the valuation ring of the place $\varphi$ to be constructed must coincide with $\mathfrak{D}=\mathfrak{D}(K / k, \tau)$. Now, by the assumption about $k^{\prime}$, the residue class ring $\mathfrak{v} / \mathrm{m}$ of $\mathfrak{D}$ is algebraic over $k$. Thus it follows from a well known theorem of Artin and Schreier [AS, Satz 8] that there exists a unique order preserving homomorphism $\beta: \mathfrak{D} / \mathrm{m} \rightarrow R$ with respect to $\bar{\tau}$ and $\varrho$ which extends $\chi$ (cf [ $\mathrm{K}_{1}$, Cor. 5.1], where this is proved by similar methods as are used in the present paper). By Proposition 1.3 the place which has the valuation ring $\mathfrak{D}$ and induces on $\mathfrak{D} / \mathrm{m}$ the map $\beta$ fulfills the conditions (i) and (ii) and is the only $R$-valued place with these properties. q.e.d.

From Proposition 1.3 and Corollary 1.5 we obtain

THEOREM 1.6. (cf [L], Th. 6). Assume that $L$ is an algebraic field extension of $K$, that $\tau$ is a signature of $L$ and that $\varphi$ is an $R$-valued place of $K$, compatible with $\tau \mid K$. Then there exists a unique $R$-valued place $\psi$ of $L$ extending $\varphi$ and compatible with $\tau$.

Proof. We chose a maximal subfield $k$ of $K$ on which $\varphi$ is trivial. Then $\varphi$ is zero dimensional over $k$. By Example 1.4 the valuation ring $\mathfrak{D}$ of $\varphi$ coincides with the valuation ring $\mathfrak{D}(K / k, \sigma)$ associated with the restriction $\sigma$ of $\tau$ to $K$. Since $L / K$ is algebraic, the residue class ring of $\mathfrak{v}(L / k, \tau)$ is algebraic over the residue class ring of $\mathfrak{D}(K / k, \sigma)$. Thus the algebraic closure $k^{\prime}$ of $k$ in $L$ is maximal archimedian with respect to $\tau$. Now we can apply Cor. 1.5 to $\sigma$ and the homomorphism $\chi=\varphi \mid k$ from $k$ to $R$ and also to 
$\tau$ and $\chi$. Clearly $\varphi$ is the place of $K$ corresponding to $\sigma$ and $\chi$ in the sense of Cor. 1.5. We denote by $\psi$ the place of $L$ corresponding to $\tau$ and $\chi$. Since $\psi \mid K$ must correspond to $\sigma$ and $\chi$ we have $\psi \mid K=\varphi$. On the other hand any $R$-valued extension $\psi^{\prime}$ of $\varphi$ to $L$ is zero dimensional over $k$. Thus if $\psi^{\prime}$ is compatible with $\tau$, Cor. 1.5 yields $\psi^{\prime}=\psi$.

\section{$\S 2$}

We want to study the orderings of a field $K$ which lie over a given $R$-valued place of $K$. For this purpose we first consider more generally an arbitrary valuation ring $\mathfrak{D}$ with maximal ideal $\mathfrak{m}$, residue class field $k=\mathfrak{v} / \mathfrak{m}$, and quotient field $K$. For any $a$ in $\mathfrak{D}$ we denote by $a$ the image in $k$. The following proposition has been proved in this generality in $\left[\mathrm{K}_{3}, \S 3\right]$ ( $\mathrm{cf}[\mathrm{Sp}],[\mathrm{M}$, Chap. $\mathrm{V}]$ if $\mathfrak{o}$ is discrete, and $[\mathrm{K}, \S 12]$ if $\mathfrak{o}$ has rank one).

PROPOSITION 2.1. There exists a unique additive map $\partial: W(K) \rightarrow W(k)$ such that $\partial(a)=(\bar{a})$ for every $a$ in $\mathrm{D}^{*}$ and $\partial(a)=0$ for every $(a)$ in $Q(K)$ which lies not in $Q(\mathfrak{D})$, i.e. with $a K^{* 2} \cap \mathfrak{D}^{*}$ empty. ${ }^{2}$

Let $v: K^{*} \rightarrow \Gamma$ denote a valuation corresponding to $\mathrm{D}$ with value group $\Gamma$. This valuation induces a map $\tilde{v}$ from $Q(K)=K^{*} / K^{* 2}$ to $\Gamma / 2 \Gamma$. We chose a subgroup $M$ of $Q(K)$ such that $\tilde{v}$ gives a bijection from $M$ to $\Gamma / 2 \Gamma$. Such a subgroup $M$ clearly exists, since $Q(K)$ and $\Gamma / 2 \Gamma$ are vector spaces over the field of two elements. We call $M$ a group of representatives for $\Gamma / 2 \Gamma$. Any element $z$ of $W(K)$ can be written - possibly in different ways - in the form $z=\sum_{m \in M} x_{m} m$ with $x_{m}$ in $W(\mathfrak{D})$ and only finitely many $x_{m} \neq 0$, since this is true for the generators $(a)$ of $W(K)$. We denote for any $x$ in $W(\mathfrak{b})$ by $\bar{x}$ the image under the natural map from $W(\mathfrak{p})$ to $W(k)$. Then one immediately computes for $m$ in $M$ :

$$
\partial(m z)=\bar{x}_{m} .
$$

In particular $\partial(m z)$ is zero for nearly all $m$ in $M$. Thus we have a map

$$
\Delta: W(K) \rightarrow W(k)[M]
$$

into the group ring of $M$ over $W(k)$, defined by

$$
\Delta(z)=\sum_{m \in M} \partial(m z) m \text {. }
$$

It is clear from (2.2) that $\Delta$ is a ring homomorphism which is surjective, since the

2) In [K $\mathrm{K}_{3}$ this map $\partial$ is denoted by $\varphi_{*}$ with $\varphi$ the place $K \rightarrow k \cup \infty$ corresponding to $\mathfrak{v}$. In the present paper $\varphi_{*}$ will have a slightly different meaning. 
natural map $W(\mathfrak{o}) \rightarrow W(k)$ is surjective. It is further clear from (2.2) that $\Delta(z)=0$ if and only if all $x_{m}$ lie in the kernel of $W(\mathfrak{o}) \rightarrow W(k)$, which we denote by $W(\mathfrak{o}, \mathrm{m})$. Summarizing we obtain

PROPOSITION 2.4. $\Delta: W(K) \rightarrow W(k)[M]$ is a ring-epimorphism whose kernel is the ideal of $W(K)$ generated by $W(\mathrm{o}, \mathrm{m})$.

Remarks. i) It has been shown in $[\mathrm{K}, \S 12]$ that the set $W(\mathfrak{D}, \mathrm{m})$ itself is an ideal of $W(K)$ if $\mathrm{o}$ has rank one.

ii) It is not difficult to prove for any local ring $\mathfrak{v}$ with maximal ideal $m$ that $W(\mathfrak{D}, \mathfrak{m})$ is generated as an ideal by the elements $1-(1+d)$ with $d$ in $\mathrm{m}$. We shall not need this fact.

We now consider a real place $\varphi: K \rightarrow R \cup \infty$ and denote by $\mathrm{D}$ the valuation ring of $\varphi$. We continue to use the notations $\mathrm{m}, k, v, \Gamma, M$ with respect to $\mathfrak{D}$ as above. $\varphi$ induces a homomorphism $\bar{\varphi}$ from $k$ into $R$. The composite map

$$
\varphi_{*}=\varrho \circ W(\bar{\varphi}) \circ \hat{\sigma}: W(K) \rightarrow W(k) \rightarrow W(R) \rightarrow \mathbb{Z}
$$

has the description given in the introduction, and the restriction of $\varphi_{*}$ to $W(\mathrm{D})$ is the ring homomorphism $\hat{\varphi}$ considered in $\S 1$. As an easy consequence of Proposition 2.4 we obtain

THEOREM 2.5. The signatures $\sigma: W(K) \rightarrow Z$ lying over a given place $\varphi: K \rightarrow R \cup \infty$ correspond uniquely to the characters $\chi: M \rightarrow\{ \pm 1\}$ by the following formulas:

$$
\chi(m)=\sigma(m)
$$

for $m$ in $M$, and

$$
\sigma(z)=\sum_{m \in M} \chi(m) \varphi_{*}(m z)
$$

for $z$ in $W(K)$.

Remark. Except the last formula this has already been proved by Krull in a different way [Kr, p. 189].

Proof. Clearly $\hat{\varphi}$ vanishes on $W(\mathrm{o}, \mathrm{m})$. Thus any signature $\sigma$ lying over $\varphi$ must vanish on the ideal generated by $W(\mathrm{o}, \mathrm{m})$ in $W(K)$. By Prop. 2.4 such a signature must have the form $\sigma=\alpha \circ \Delta$ with a uniquely determined ring homomorphism $\alpha$ from $W(k)[M]$ to $\mathbf{Z}$. These homomorphisms $\alpha$ correspond uniquely to the pairs $\left(\alpha_{0}, \chi\right)$ consisting of a homomorphism $\alpha_{0}: W(k) \rightarrow \mathbf{Z}$ and a character $\chi: M \rightarrow\{ \pm 1\}$, the correspondence being given by $\alpha_{0}=\alpha \mid W(k)$ and $\chi=\alpha \mid M$. From the definition (2.3) of $\Delta$ we obtain for the signature $\sigma=\alpha \circ \Delta$ and $z$ in $W(K)$ :

$$
\sigma(z)=\sum_{m \in M} \chi(m)\left(\alpha_{0} \circ \partial\right)(m z) .
$$


Such a signature $\sigma$ coincides with $\hat{\varphi}$ on $W(\mathfrak{D})$ if and only if $\alpha_{0}(\bar{z})=\hat{\varphi}(z)$ for all $z$ in $W(\mathfrak{b})$. This means $\alpha_{0} \circ \partial=\varphi_{*}$. Theorem 2.5 is now obvious. q.e.d.

THEOREM 2.6. Let $L$ be an arbitrary field extension of $K$ and $\psi: L \rightarrow R \cup \infty a$ real place. Further let $\sigma$ be a signature of $K$. Then the following are equivalent:

(i) There exists a signature $\tau$ of $L$ which lies over $\psi$ and extends $\sigma$.

(ii) $\sigma(a)=\psi_{*}\left(\right.$ a) for all $a$ in $K^{*}$ such that $\psi_{*}(a) \neq 0$.

Proof. (i) $\Rightarrow$ (ii) is evident. We now assume (ii), which in particular implies that $\sigma$ lies over the restriction $\varphi=\psi \mid K$. We denote by $\mathcal{D}$ and $\mathcal{D}$ the valuation rings of $\varphi$ resp. $\psi$, and by $\overline{\mathbf{D}}$ and $\mathfrak{D}$ their residue class fields, further by $w^{\prime}: L^{*} \rightarrow \Gamma^{\prime}$ a valuation corresponding to $\mathfrak{D}$ with value group $\Gamma^{\prime}$ and by $\Gamma$ the value group $w^{\prime}\left(K^{*}\right)$ of $w \mid K^{*}$. We chose a group $M$ of representatives for $\Gamma / 2 \Gamma$ in $Q(K)$. Let $M_{0}$ denote the subgroup of all $m$ in $M$ with $w^{\prime}(m)$ in $2 \Gamma^{\prime}$, and let $M_{1}$ denote an arbitrary chosen subgroup of $M$ such that $M=M_{0} \times M_{1}$. The map from $M_{1}$ into $\Gamma^{\prime} / 2 \Gamma^{\prime}$ induced by $w$ is injective. Thus also the natural map from $M_{1}$ into $Q(L)$ is injective, and we can chose a group $N$ of representatives of $\Gamma^{\prime} / 2 \Gamma^{\prime}$ in $Q(L)$ which contains the image $N_{1}$ of $M_{1}$. We further chose a subgroup $N_{0}$ of $N$ such that $N=N_{0} \times N_{1}$. Finally let $\Delta$ denote the map (2.3) from $W(K)$ onto $W(\overline{\mathfrak{D}})[M]$ and $\Delta^{\prime}$ the analogous map from $W(L)$ onto $W(\bar{D})[N]$.

By the proof of Theorem 2.5 we have $\sigma=\alpha \circ \Delta$ with a homomorphism $\alpha$ from $W(\overline{\mathfrak{D}})[M]$ to $\mathbf{Z}$ which extends the homomorphism $\bar{\varphi}_{*}=\varrho \circ W(\bar{\varphi})$ from $W(\overline{\mathfrak{D}})$ to $\mathbf{Z}$. Similarly $\tau$ must have the form $\tau=\beta \circ \Delta^{\prime}$ with a homomorphism $\beta$ from $W(\mathfrak{D})[N]$ to $\mathbf{Z}$ which we have to construct. Since the natural map from $Q(K)$ to $Q(L)$ maps $M_{0}$ into $Q(\mathfrak{D}) \subset W(\mathfrak{D})$, there is an obvious map $\lambda$ from the ring $R:=W(\overline{\mathfrak{b}})\left[M_{0}\right]$ to the ring $S:=W(\mathfrak{D})$. Combining $\lambda$ with the bijection $M_{1} \simeq N_{1}$ induced by $Q(K) \rightarrow Q(L)$ we obtain a map $\mu$ from the ring $R\left[M_{1}\right]=W(\bar{b})[M]$ to $S\left[N_{1}\right]$. Consider now the diagram

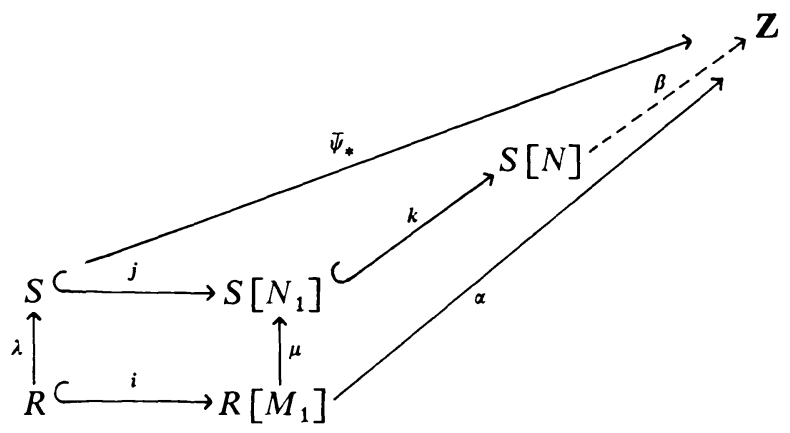

with inclusion maps $i, j, k$. That $\tau$ lies over $\psi$ means that the upper triangle is commutative, and that $\tau$ extends $\sigma$ means that the lower triangle is commutative. Our hypothesis (ii) means $\bar{\psi}_{*} \circ \lambda=\alpha \circ i$. Now the square with the arrows $i, \mu, \lambda, j$ is a pushout 
\{i.e. gives a description of $S\left[N_{1}\right]$ as the tensor product of $S$ and $R\left[M_{1}\right]$ over $R$. Thus there is a unique map $\gamma: S\left[N_{1}\right] \rightarrow \mathbf{Z}$ with $\gamma \circ j=\bar{\psi}_{*}$ and $\gamma \circ \mu=\alpha$. The only condition which $\beta$ has to fulfill is the commutativity of the following triangle:

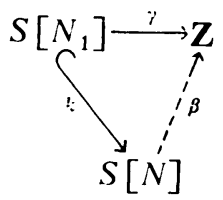

Since $S[N]=S\left[N_{1}\right]\left[N_{0}\right]$ clearly such a homomorphism $\beta$ exists. q.e.d.

Remark 2.7. More precisely the extensions of $\gamma: S\left[N_{1}\right] \rightarrow \mathbf{Z}$ to $\mathbf{Z}$-valued homomorphisms $\beta$ of $S[N]$ correspond bijectively to the characters of $N_{0}$. The map from $N_{0}$ to $\Gamma^{\prime} /\left(2 \Gamma^{\prime}+\Gamma\right)$ induced by $w^{\prime}$ is bijective. Thus the signatures $\tau$ of $L$ extending $\sigma$ and lying over $\psi$ correspond bijectively to the characters of $\Gamma^{\prime} /\left(2 \Gamma^{\prime}+\Gamma\right)$ (in a noncanonical way), if there are any such signatures $\tau$.

We close this section with an application of the last two theorems.

THEOREM 2.8. Assume that $L_{1}$ and $L_{2}$ are field extensions of a field $K$ and that $L_{1}$ is algebraic over $K$. Further assume that on each $L_{i}$ an $R$-valued place $\varphi_{i}$ is given such that $\varphi_{1}$ and $\varphi_{2}$ coincide on $K$. Then the following are equivalent:

(i) There exists a field composite $F$ of $L_{1}$ and $L_{2}$ over $K$ and an $R$-valued place $\psi$ on $F$ extending both $\varphi_{1}$ and $\varphi_{2}$.

(ii) $\varphi_{1 *}(a)=\varphi_{2 *}$ (a) for all $a$ in $K^{*}$ such that both $\varphi_{1 *}(a)$ and $\varphi_{2 *}(a)$ are not zero.

Proof. (i) $\Rightarrow$ (ii) is trivial. We now assume that (ii) holds. We first construct on each $L_{i}$ a signature $\sigma_{i}$ lying over $\varphi_{i}$ such that $\sigma_{1}\left|K=\sigma_{2}\right| K$ : Let $\varphi$ denote the restriction $\varphi_{1}\left|K=\varphi_{2}\right| K$, let $\Gamma$ denote the value group of a valuation of $K$ corresponding to $\varphi$, and $M$ denote a group of representatives of $\Gamma / 2 \Gamma$ in the group $Q(K)$. Further let $A_{i}(i=1,2)$ denote the subgroup of all $m$ in $M$ such that $\varphi_{i *}(m) \neq 0$ and let $\chi_{i}$ denote the character $\varphi_{i *} \mid A_{i}$ of $A_{i}$. By hypothesis $\chi_{1}$ and $\chi_{2}$ coincide on $A_{1} \cap A_{2}$. Thus it is possible to choose a character $\chi$ of $M$ with $\chi \mid A_{i}=\chi_{i}$ for $i=1,2$. Let $\sigma$ denote the signature of $K$ lying over $\varphi$ and corresponding to the character $\chi$ as explained in Theorem 2.5. Clearly $\sigma(m)=\varphi_{1 *}(m)$ for any $m$ in $M$ with $\varphi_{1 *}(m) \neq 0$ and thus $\sigma(a)=\varphi_{1 *}(a)$ for any $a$ in $K^{*}$ with $\varphi_{1 *}(a) \neq 0$. The same holds with $\varphi_{2}$ instead of $\varphi_{1}$. By Theorem 2.6 there exists a signature $\sigma_{i}$ on each $L_{i}$ which lies over $\varphi_{i}$ and extends $\sigma$.

We now obtain a field composite $F$ of $L_{1}$ and $L_{2}$ over $K$ and a signature $\tau$ on $F$ extending both $\sigma_{1}$ and $\sigma_{2}$ in the following way: Let $S$ be a real closure of $L_{2}$ with respect to $\sigma_{2}$ and $\gamma$ denote the signature of $S$. There exists a (unique) homomorphism $f: L_{1} \rightarrow S$ over $K$ which is compatible with $\sigma_{1}$ and $\gamma$, i.e. $\sigma_{1}=\gamma \circ W(f)$. \{Apply [AS], Satz 8. This is also a special case of our Theorem 1.6: Extend the trivial place $K \hookrightarrow S$ 
to an $S$-valued place on $L_{1}$ compatible with $\sigma_{1}$. $\}$ The field composite $F:=f\left(L_{1}\right) L_{2} \subset S$ and the signature $\tau:=\gamma \mid F$ have the desired properties.

By Theorem 1.6 there is a unique $R$-valued place $\psi$ on $F$ which extends $\varphi_{2}$ and is compatible with $\tau$. Clearly $\psi \mid L_{1}$ is compatible with $\sigma_{1}$ and extends $\varphi$. Thus by the same theorem $\psi \mid L_{1}=\varphi_{1}$. q.e.d.

Remark 2.9. In the proof just completed we used the fact that for given signatures $\sigma_{1}, \sigma_{2}$ on $L_{1}$ and $L_{2}$ with $\sigma_{1}\left|K=\sigma_{2}\right| K$ there exists a field composite $F$ of $L_{1}$ and $L_{2}$ over $K$ and a signature $\tau$ on $F$ with $\tau \mid L_{1}=\sigma_{1}$ and $\tau \mid L_{2}=\sigma_{2}$. This remains true if both $L_{1}$ and $L_{2}$ are arbitrary field extensions of $K$ with $F$ a free field composite. Further it can be shown that for given $\sigma_{1}$ and $\sigma_{2}$ up to equivalence only one such free composite $F$ and only one such $\tau$ exists. These facts are closely related to the following theorem (see also $\left[\mathrm{K}_{2}\right.$, Th. 3]): Denote by $A$ the total quotient ring of $L_{1} \otimes_{K} L_{2}$. The kernel and the cokernel of the obvious map from $W\left(L_{1}\right) \otimes_{W(K)} W\left(L_{2}\right)$ to $W(A)$ are 2-primary torsion groups. I omit the proofs since we do not need these results in the present paper.

In the situation of Theorem 3.9 it may happen that there exists more than one $R$-valued place $\psi$ on a field composite $F$ of $L_{1}$ and $L_{2}$ which extends both $\varphi_{1}, \varphi_{2}$, as shows the following

EXAMPLE 2.10. Let $\varphi$ be an $R$-valued place on a field $K$ and let $a$ and $c$ be elements of $K$ such that $\varphi_{*}(a)=0, \varphi_{*}(c)=1, c$ not a square. For example let $K=R(t)$ with one indeterminate $t$, let $\varphi$ be the place over $R$ with $\varphi(t)=0$ and $a=t, c=1+t^{2}$. Using the trace formula proved in the next section (see also Introduction) one easily checks that there is exactly one $R$-valued place $\varphi_{1}$ on $L_{1}:=K(\sqrt{ } a)$ and one $R$-valued place $\varphi_{2}$ on $L_{2}:=K(\sqrt{a c})$ which extend $\varphi$ (Of course this also follows from general valuation theory). The field $F=K(\sqrt{a}, \sqrt{a c})$ is the only composite of $L_{1}$ and $L_{2}$ over $K$. But $\varphi_{2}$ has - by the same trace formula - exactly two extensions $\psi, \psi^{\prime}$ to $F$ with values in $R$, which both must also extend $\varphi_{1}$.

Assume that $L$ is a finite extension of degree $n$ of a field $K$ and that an $R$-valued place $\varphi$ is given on $K$. Let $\operatorname{Tr}^{*}: W(L) \rightarrow W(K)$ denote the transfer map from $W(L)$ to $W(K)$ with respect to the regular trace $\operatorname{Tr}=\operatorname{Tr}_{L / K}$ ([S], cf. Introduction). The goal of this section is to prove the following trace formula:

THEOREM 3.1. For every $x$ in $W(L)$

$$
\varphi_{*}\left(\operatorname{Tr}^{*}(x)\right)=\sum_{\psi\lceil\varphi} \psi_{*}(x) \text {, }
$$

with the sum taken over all $R$-valued places $\psi$ on $L$ which extend $\varphi$. 
N.B. The sum is finite, since $\varphi$ has at most $n$ extensions $\psi$ to L [B, Chap VI, $\S 8$ no. 3, Th. 1].

We shall deduce this theorem from our results about the connection between signatures and real places in the previous sections and from the following trace formula for signatures, which is a consequence of Artin-Schreier's theory of real closures (see $\left.\left[K_{1}, \S 5\right]\right)$ :

PROPOSITION 3.2. For every signature $\sigma$ of $K$ and every element $x$ of $W(L)$

$$
\sigma\left(\operatorname{Tr}^{*}(x)\right)=\sum_{\tau \mid \sigma} \tau(x),
$$

where $\tau$ runs through the finite set of all signatures of $L$ which extend $\sigma$

Let $\psi_{i}, 1 \leqslant i \leqslant r$, denote the $R$-valued places of $L$ which extend $K(r=0$, if there are no such places). We chose a valuation $v: K^{*} \rightarrow \Gamma$ with value group $\Gamma$ corresponding to the place $\varphi$, and for each $\psi_{i}, 1 \leqslant i \leqslant r$, a corresponding valuation $w_{i}: L^{*} \rightarrow \Gamma_{i}$ extending $v$ with value group $\Gamma_{i} \supset \Gamma$. Since $\sum_{i}\left(\Gamma_{i}: \Gamma\right) \leqslant n$ [B, loc. cit.], all $\left(\Gamma_{i}: \Gamma\right)$ are finite.

To prove Theorem 3.1 we have to surmount some technical difficulties, which arise from the fact that $\Gamma$ may not be finitely generated. To get an idea of the proof the reader is advised to follow first the proof under the additional assumption that $\Gamma$ is finitely generated. Then it is clear (and follows from Lemma 3.3 below), that $\Gamma / 2 \Gamma$ and all $\Gamma_{i} / 2 \Gamma_{i}$ have the same finite cardinality. The proof goes through with the choice $M_{0}=M, M_{1}=\{1\}, N_{i 0}=N_{i}$ below, and Lemma 3.3 and 3.4 may be skipped.

LEMMA 3.3. For each $i$ with $1 \leqslant i \leqslant r$ the kernel and the cokernel of the natural map $\alpha_{i}: \Gamma / 2 \Gamma \rightarrow \Gamma_{i} / 2 \Gamma_{i}$ are finite and have the same cardinality.

Proof. Consider the commutative diagram

$$
\begin{aligned}
& 0 \rightarrow \rightarrow \Gamma_{i} \rightarrow \Gamma_{i} / \Gamma \rightarrow 0 \\
& \downarrow \downarrow^{2} \downarrow^{2} \quad \downarrow^{2} \\
& 0 \rightarrow \Gamma \rightarrow \Gamma_{i} \rightarrow \Gamma_{i} / \Gamma \rightarrow 0
\end{aligned}
$$

of exact sequences, where the vertical arrows denote the homotheties $x \mapsto 2 x$. Since $\Gamma_{i} / \Gamma$ is finite, the kernel $A_{i}$ and the cokernel $B_{i}$ of $\Gamma_{i} / \Gamma^{2} \rightarrow \Gamma_{i} / \Gamma$ have the same finite cardinality. Now the snake lemma gives an exact sequence

$$
0 \rightarrow A_{i} \rightarrow \Gamma / 2 \Gamma \stackrel{\alpha_{i}}{\rightarrow} \Gamma_{i} / 2 \Gamma_{i} \rightarrow B_{i} \rightarrow 0
$$

which makes the assertion obvious. q.e.d.

In $Q(K) \cong K^{*} / K^{* 2}$ we chose a group $M$ of representatives of $\Gamma / 2 \Gamma$. Then after fixing an element $x$ of $W(L)$ we chose a decomposition $M=M_{0} \times M_{1}$ with the following properties: 
a) $M_{0}$ is finite,

b) $M_{0}$ contains the representatives of the elements of all finite subsets $\operatorname{Ker} \alpha_{i}$, $1 \leqslant i \leqslant r$, of $\Gamma / 2 \Gamma$,

c) $\varphi_{*}\left(m \cdot \operatorname{Tr}^{*}(x)\right)=0$ for $m$ in $M$ but not in $M_{0}$,

d) $\psi_{i *}(m \cdot x)=0$ for $1 \leqslant i \leqslant r$ and all $m$ in $M_{1}$ with $m \neq 1$.

Clearly such a decomposition of $M$ does exist. By property b) the map $Q(K) \rightarrow Q(L)$ is injective on $M_{1}$. Thus we regard $M_{1}$ also as a subgroup of $Q(L)$. By property b) further all maps $w_{i}: Q(L) \rightarrow \Gamma_{i} / 2 \Gamma_{i}$ are injective on $M_{1}$. For each $i$ with $1 \leqslant i \leqslant r$ we chose a group $N_{i}$ of representatives of $\Gamma_{i} / 2 \Gamma_{i}$ in $Q(L)$ which contains $M_{1}$. Then we chose a decomposition $N_{i}=N_{i 0} \times M_{1}$.

LEMMA 3.4. All $N_{i 0}, 1 \leqslant i \leqslant r$, have the same cardinality as $M_{0}$.

Proof. For each $i, 1 \leqslant i \leqslant r$, we have a commutative diagram of exact sequences

$$
\begin{aligned}
0 & \rightarrow \tilde{v}\left(M_{1}\right) \rightarrow \Gamma / 2 \Gamma \rightarrow C \\
\downarrow & \rightarrow 0 \\
0 & \rightarrow \tilde{w}_{i}\left(M_{1}\right) \rightarrow \Gamma_{i} / 2 \Gamma_{i} \rightarrow D_{i} \rightarrow 0
\end{aligned}
$$

with groups $C$ and $D_{i}$ which have the cardinalities $|C|=\left|M_{0}\right|$ and $\left|D_{i}\right|=\left|N_{i 0}\right|$. Let $X_{i}$ denote the kernel and $Y_{i}$ the cokernel of the map $C \rightarrow D_{i}$. Clearly the map $\tilde{v}\left(M_{1}\right) \rightarrow$ $\rightarrow \tilde{w}_{i}\left(M_{1}\right)$ is bijective. Thus $X_{i} \cong \operatorname{Ker} \alpha_{i}$ and $Y_{i} \cong \operatorname{Coker} \alpha_{i}$. Lemma 3.4 now follows from Lemma 3.3 and the exact sequence

$$
0 \rightarrow X_{i} \rightarrow C \rightarrow D \rightarrow Y_{i} \rightarrow 0 \text {. q.e.d. }
$$

After these preparations we consider sets $S$ and $T_{i}, 1 \leqslant i \leqslant r$, of signatures, defined as follows: $S=$ set of all signatures $\sigma$ of $K$ lying over $\varphi$ with $\sigma(m)=1$ for all $m$ in $M_{1}$; further $T_{i}=$ set of all signatures $\tau$ of $L$ lying over $\psi_{i}$ with $\tau(m)=1$ for all $m$ in $M_{1}$. We regard the group $\hat{M}_{0}$ of characters of $M_{0}$ as the subgroup of characters of $M$ which are trivial on $M_{1}$, and $\hat{N}_{i 0}$ as the group of characters of $N_{i}$ which are trivial on $M_{1}$. Theorem 2.5 gives a bijection from $S$ to $\hat{M}_{0}$ mapping each $\sigma$ in $S$ to the corresponding character of $M$, and in the same way a bijection from $T_{i}$ to $\widehat{N}_{i 0}$. Thus we know from Lemma 3.4 that the sets $S, T_{i}, 1 \leqslant i \leqslant r$, are all finite and have the same cardinality $\left|M_{0}\right|$.

Now Theorem 1.6 says, that for each signature $\tau$ of $L$, whose restriction $\tau \mid K$ lies over $\varphi$, there exists a unique $R$-valued place $\psi$ of $L$ which extends $\varphi$ and is compatible with $\tau$. Thus the union $T=\bigcup_{i} T_{i}$ of all $T_{i}\{T=\emptyset$ if $r=0\}$ is the set of all signatures $\tau$ of $L$ whose restrictions $\tau \mid K$ belong to $S$, and furthermore this union is disjoint, $T_{i} \cap T_{j}=\emptyset$ for $i \neq j$.

Theorem 3.1 will now come out by computing the finite sum $\sum_{\tau \in T} \tau(x)$ with our 
fixed element $x$ in two different ways. On the one hand,

$$
\sum_{\tau \in T} \tau(x)=\sum_{\sigma \in S} \sum_{\tau \mid \sigma} \tau(x)
$$

Thus by Prop. 3.2 with $z=\operatorname{Tr}^{*}(x)$ :

$$
\sum_{\tau \in T} \tau(x)=\sum_{\sigma \in S} \sigma(z) .
$$

By the last formula in Theorem 2.5 this sum equals

$$
\sum_{\chi \in \hat{\mathbf{M}}_{0}}\left(\sum_{m \in M} \chi(m) \varphi_{*}(m z)\right)
$$

By the property c) of the decomposition $M=M_{0} \times M_{1}$ we may replace $M$ by $M_{0}$ in the interior sum. Then interchanging the summations we obtain

$$
\sum_{\tau \in T} \tau(x)=\left|M_{0}\right| \varphi_{*}(z) .
$$

On the other hand

$$
\sum_{\tau \in T} \tau(x)=\sum_{i=1}^{r} \sum_{\tau \in T_{i}} \tau(x)
$$

$\{$ Read zero for the right hand side if $r=0\}$. Again by Theorem 2.5

$$
\sum_{\tau \in T_{i}} \tau(x)=\sum_{\chi \in N_{i 0}}\left(\sum_{m \in N_{i}} \chi\left(m_{0}\right) \psi_{i *}(m x)\right),
$$

where $m_{0}$ denotes the component of $m$ in $N_{i 0}$. Since $N_{i 0}$ is finite, we may interchange the summations and obtain for the right hand side

$$
\sum_{m \in N_{t}} \psi_{i *}(m x)\left(\sum_{\chi \in N_{i 0}} \chi\left(m_{0}\right)\right) \text {. }
$$

The interior sum is zero if $m_{0} \neq 1$. But if $m_{0}=1$, then $m \in M_{1}$ and by property d) of our decomposition of $M$ the factor $\psi_{i *}(m x)$ vanishes except for $m=1$. Thus this sum reduces to $\left|N_{i 0}\right| \psi_{i *}(x)$, and we obtain

$$
\sum_{\tau \in T} \tau(x)=\sum_{i=1}^{r}\left|N_{i 0}\right| \psi_{i *}(x) .
$$

Theorem 3.1 now follows from (3.5), (3.6), and Lemma (3.4).

If $\varphi$ is a discrete place, a more direct and more geometric proof of Theorem 3.1 without resorting to signatures follows easily from the lemma on p. 322 in [G]. It would be desirable to have a similar proof in the general case. The main difficulty 
seems to be that one has to work with quadratic forms on modules, which in general are not finitely generated.

This section does not use the content of $\S 2$ and $\S 3$. Assume that $\varphi: K \rightarrow R \cup \infty$ is a place from a field $K$ to a real closed field $R$ and that $L$ is a finitely generated field extension of $K$. We ask for a criterion that $\varphi$ is extendable to an $R$-valued place of $L$. Let $\mathfrak{D}$ denote the valuation ring of $\varphi$ and as in $\S 1$ let $\hat{\varphi}$ denote the homomorphism from $W(\mathbf{D})$ to $\mathbf{Z}$ induced by $\varphi$.

PROPOSITION 4.1. $\varphi$ is extendable to an $R$-valued place of $L$ if and only if $\hat{\varphi}$ vanishes on the kernel of the natural map $W(\mathfrak{D}) \rightarrow W(L)$.

Proof. We denote this kernel by $W(\mathrm{D}, L)$.

i) Assume that $\psi: L \rightarrow R \cup \infty$ is an extension of $\varphi$. Then for every $Z$ in $W(\mathfrak{o})$ clearly $\hat{\varphi}(Z)=\hat{\psi}\left(Z_{L}\right)$ with $Z_{L}$ the image of $Z$ in $W(L)$ under the natural map $W(K) \rightarrow W(L)$. Thus $\hat{\varphi}(Z)=0$ if $Z$ is in $W(\mathfrak{o}, L)$.

ii) Now assume $\hat{\varphi}(W(\mathfrak{D}, L)=0$. We first construct a signature $\tau$ of $L$ such that $\tau \mid K$ lies over $\varphi$. We proceed as in the proof of Prop. 1.1. The kernel $P$ of the ringhomomorphism $\hat{\varphi}$ from $W(\mathfrak{D})$ onto $\mathbf{Z}$ contains $W(\mathfrak{D}, L)$ and thus yields a prime ideal $\bar{P}$ of $W(\mathfrak{D}) / W(\mathfrak{D}, L)$, which is a subring of $W(L)$ in a natural way. $\bar{P}$ must be minimal [KRW]. Thus we can find a minimal prime ideal $Q$ of $W(L)$ lying over $\bar{P}$. Since $W(\mathfrak{o}) / P \cong \mathbf{Z}$ embeds into $W(L) / Q$ we have $W(L) / Q \cong \mathbf{Z}$. The only homomorphism $\tau: W(L) \rightarrow \mathbf{Z}$ with kernel $Q$ is a signature whose restriction $\sigma=\tau \mid K$ lies over $\varphi$.

Let $S$ be a real closure of $L$ with respect to $\tau$. Then the algebraic closure $K^{\prime}$ of $K$ in $S$ is a real closure of $K$ with respect to $\sigma$. By Theorem 1.6 there exists a unique $R$-valued place $\gamma$ of $K^{\prime}$ which extends $\varphi$. On the other hand the composite field $L^{\prime}=L K^{\prime}$ in $S$ is a finitely generated formally real field extension of $K^{\prime}$. Thus by a well known theorem of Artin and Lang ([L, Theorem 7], see also $\left.\left[K_{1}, \S 6\right]\right)$ there exists a place $\chi: L^{\prime} \rightarrow K^{\prime} \cup \infty$ which is the identity on $K^{\prime}$. The $R$-valued place $\gamma \circ \chi$ on $L^{\prime}$ extends $\gamma$ and thus its restriction to $L$ extends $\varphi$. q.e.d.

For any field extension $L / K$ we denote by $W(K, L)$ the kernel of the natural map from $W(K)$ to $W(L)$.

THEOREM 4.2. Let $L \supset K \supset k$ be three fields such that $L / k$ is finitely generated. Then the following are equivalent:

i) Any place $\varphi: K \rightarrow R \cup \infty$ into a real closed field $R$ which is trivial on $k$ can be extended to an $R$-valued place of $L$.

ii) The statement i) with the additional condition inserted, that $R$ is algebraic over $\varphi(k)$. 
iii) $W(K, L)$ is a torsion group.

Notice that in statement (iii) the field $k$ does not occur.

Proof. (i) $\Rightarrow$ (ii) is trivial, and (iii) $\Rightarrow$ (i) follows from the previous proposition 4.1. To prove (ii) $\Rightarrow$ (iii) we assume that there exists an element $Z$ in $W(K, L)$ which is not torsion. We have to show that there exists a real closure $R$ of $k$ and a place $\varphi: K \rightarrow$ $\rightarrow R \cup \infty$ which is the identity on $k$, such that $\varphi$ cannot be extended to an $R$-valued place of $L$. We write

$$
Z=\sum_{i=1}^{n}\left(a_{i}\right)
$$

with elements $\left(a_{i}\right)$ in $Q(K)$. Since $Z$ is not a torsion element there exists a signature $\sigma$ of $K$ such that

$$
\sigma(Z)=\sum_{i=1}^{n} \sigma\left(a_{i}\right) \neq 0
$$

([P, Satz 22], see also [LL], [KRW]). Let $S$ denote a real closure of $K$ with respect to $\sigma$ and $R$ denote the algebraic closure of $k$ in $S$. Finally let $K^{\prime}$ denote the field composite $K R$ in $S$, which is finitely generated over $R$. According to Artin and Lang [L, Th. 8, p. 387] there exists a place $\gamma: K^{\prime} \rightarrow R \cup \infty$, which is the identity on $R$, such that all $\gamma\left(a_{i}\right), 1 \leqslant i \leqslant n$ are finite and not zero and $\hat{\gamma}\left(a_{i}\right)=\sigma\left(a_{i}\right)$. Let $\varphi$ denote the restriction of $\gamma$ to $K$ and $\mathfrak{D}$ the valuation ring of $\varphi$. Clearly $Z \in W(\mathfrak{o})$ and $\hat{\varphi}(Z)=\sigma(Z) \neq 0$. By Proposition 4.1 this place $\varphi$ can not be extended to $L$. q.e.d.

\section{REFERENCES}

[AS] Artin, E. and SchreIer, O., Algebraische Konstruktion reeller Körper, Hamburger Abh. 5 (1926), 85-99.

[B] Bourbaki, N., Éléments de mathématique, Algèbre commutative, Hermann, Paris.

[G] Geyer, W. D., Harder, G., Knebusch, M., Scharlau, W., Ein Residuensatz für symmetrische Bilinearformen, Invent. Math. 11 (1970), 319-328.

[H] Harrison, D. K., Wittrings, Lecture notes, Dept. of Math., Univ. of Kentucky, Lexington, Kentucky, 1970.

[K] KNeBuSCH, M., Grothendieck- und Wittringe von nichtausgearteten symmetrischen Bilinearformen, Sitzber. Heidelber. Akad. Wiss. 1969/70, 3. Abhandlung, pp. 93-157 (also obtainable as separate volume from Springer Verlag).

[K $\left.\mathrm{K}_{1}\right]$ KNeBuSCH, M., On the uniqueness of real closures and the existence of real places, Comment. Math. Helv. 47 (1972), 260-269.

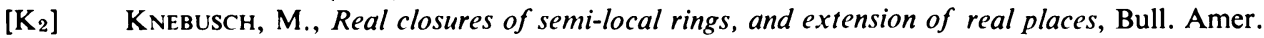
Math. Soc. 79 (1973), 78-81.

[K $\left.\mathrm{K}_{3}\right]$ KNeBusCh, M., Specialization of quadratic and symmetric bilinear forms, and a norm theorem, to appear in Acta Arithmetica 24 (1973).

[KRW] Knebusch, M., Rosenberg, A., and Ware, R., Structure of Witt-rings and quotients of abelian group rings, Amer. J. Math. 94 (1972), 119-155. 
[KWR ${ }_{1}$ ] KNebusch, M., Rosenberg, A., and WARE, R., Grothendieck- and Witt-rings of hermitian forms over Dedekind rings, Pacific J. Math. 43 (1972), 657-673.

[KWR ${ }_{2}$ ] KNebusch, M., Rosenberg, A., and Ware, R., Signatures on semi-local rings, to appear in J. of Algebra 26 (1973).

[Kr] Krull, W., Allgemeine Bewertungstheorie, J. reine angew. Math. 167 (1931), 160-196.

[L] LANG, S., The theory of real places, Annals Math. 57 (1953), 378-391.

[LL] Leicht, J. and Lorenz, F., Die Primideale des Wittschen Ringes, Invent. math. 10 (1970). $82-88$.

[M] Milnor, J., and Husemoller, D., Symmetric bilinear forms, Ergebnisse Math. 73, Springer, Berlin - Heidelberg - New York 1973.

[P] PFISTER, A., Quadratische Formen in beliebigen Körpern, Invent. math. $I$ (1966), 116-132.

[S] Scharlau, W., Zur Pfisterschen Theorie der quadratischen Formen, Invent. math. 6 (1969), 327-328.

[Sp] SPRINGER, T. A., Quadratic forms over fields with a discrete valuation I, Indag. Math. 17 (1955), 352-362.

[W] WITT, E., Theorie der quadratischen Formen in beliebigen Körpern, J. reine angew. Math. 176 (1937), 31-44.

Mathematisches Institut

der Universität des Saarlandes

BRD, 66-Saarbrücken 15

Received January 8, 1973 